\title{
An IoT-based Smart Home System for Disabled People
}

\author{
FARNAZ TAJADOD, YANYAN LI, AHMAD R. HADAEGH \\ Department of Computer Science and Information Systems \\ California State University San Marcos \\ San Marcos, CA, USA
}

\begin{abstract}
Internet of Things (IoT) envisions a future in which physical objects and the digital world can communicate. Technical communications among appropriate information can create new categories of applications and services. Today, IoT is considered a suitable infrastructure technology for low-range wireless home control systems. In fact, smart homes and buildings provide a wide range of services, applications, equipment, networks, and systems that work together to provide an intelligent environment in areas such as security, control, communications, leisure, and comfort. The purpose of this research is an implementation of an IoT-based Smart home system that provides remote access control for disabled people to open the door lock and turn on or off the lights. Besides, this IoT-based smart home system was extended with two 2-Channel Relay Modules, which assign four keys to direct different components. In addition, this system offers a safe home environment that monitored any hazardous gas leakage, smoke, and fire, or unauthorized person. The motion sensor with a dual purpose has a bright feature that enables two applications: Energy Management System and Security System. Moreover, there are three options for users to open the door for authorized people: Using the Android application on their smartphones, voice command via Google assistant, and the "Unlock door pattern." Authorized people such as friends and family, caregivers, or dog walkers, can unlock the door by physically entering the key pattern via embedded switch buttons (doorbell, B, and C). The camera in this project gets activated as soon as someone rings the doorbell; a snapshot of the current situation behind the door will be sent to the registered user with a notification email. If the entered key pattern does not match the "Unlock door pattern," the alarm system will be triggered. The significance of this research is to provide a solution to help disabled people to live as conveniently and safely as possible in their homes.
\end{abstract}

\section{Key-Words: - IoT, Smart Home Automation, Sensors, Android App, Voice Commands, Disabled People}

Received: February 28, 2021. Revised: November 29, 2021. Accepted: December 20, 2021. Published: December 31, 2021.

\section{Introduction}

A smart home or workplace can provide comfort and improve the quality of life. This process of intelligence has different dimensions and modes. People can decide what to make smart according to their needs. Smart homes with a variety of features and tools can provide a safe and peaceful place. It is difficult for people with serious chronic diseases such as sclerosis, osteoarthritis, or other movement disorders to do their daily tasks. This condition is much more difficult and distressing for those who have a disability or are unable to walk or move [1]. The idea of smart homes seems to have originated in Hollywood. In fact, a 1999 Disney movie "Smart House" shows an American family winning a house of the future with an android maid who causes havoc [2].

In this work, an IoT-based smart home system is implemented specifically for disabled people to provide a safe, comfortable environment at home.
Individuals with limited movements can open the door by touching a button on their smartphones or saying a simple command to Google assistant at their convenience. Although similar devices have been implemented in other works, we went even further to accomplish better and easier methods to move around a house. If the disabled user does not have access to his smartphone to open the door, an authorized person can unlock the door from outside by entering a "key pattern." There are several different models of smart locks with touch screens or keyless entry in the market; However, not only the average price is relatively high, but also the user may need to replace the entire door.

In addition, this proposed system provides a safe environment as it continuously monitors hazardous gas leakage and smoke. Another interesting aspect of this implementation is the multi-user feature which allows more than one person to use its appliances. This is also useful when parents consider restricting 
their children's access to the system so that they cannot open the door or change the temperature (if $\mathrm{AC}$ is the other device). Users can add their desired home appliance such as TV, AC, or any other device to control remotely since this system has one available key to get connected with any device.

Home Energy Management Systems (HEMS) have gained so much popularity worldwide that it has become one of the serious factors to reduce energy consumption. Environmental integrity, easy access, and energy consumption control are important aspects of IoT systems. Smart homes connect to a central computer via a network. This smart grid is based on information gathered in the computer's central processing about each member of a household's performed activities. It is done at different times in different parts of the house based on a software program that regulates the amount of light. In such circumstances, using digital smart home systems and providing access to more amenities for residents of smart homes can play a very important role in reducing energy costs.

In this work, we also focus on reducing energy consumption by controlling the time that lights stay on for two rooms. If a user forgets to turn off the lights, the system will turn them off after users leave.

Thanks to the Internet of Things, smart homes, and home energy management systems, disability today can no longer prevent individuals from falling behind in their daily household chores. In fact, smart homes for disabled people can bypass any movement limitations and help maintain the independence of these people. We expect that this research will be a small but effective step for helping the improvement of automation systems for disabled people and their daily interactions.

\section{Related Work}

Smart home technology has come a long way, and now, more than ever, it has automated day-to-day tasks so that we can live easier, safer, and healthier. This technology can be a powerful tool in self-care and act as an assistant by automating everything such as door locks, lights, security cameras, motion sensors, garage doors, smoke sensors, curtains, and many more. The smart building provides a wide variety of possibilities that each person can benefit from, depending on their needs [3].

According to the characteristics of the place, all tools and equipment inside a building are displayed by a user interface and can be controlled and managed by users. Given the importance of energy management in buildings and its potential for energy saving, much research and development have been devoted to optimizing energy consumption in smart buildings. Researchers have proposed control strategies that create environments that can meet the comfort needs of their users. Detecting room occupancy, receiving daylight, and turning off lights while no one is present are the most cost-effective ways to minimize the energy consumption.

Many previous similar works used different controller boards such as Arduino and Intel Galileo, while we used Raspberry Pi. Lower power requirements, less expensive, more open resources to work with, and being impossible to "brick" the pi through software are advantages of considering Raspberry pi in our implementation [4].

Other similar projects that designed a smart home for disabled individuals did not develop any user interface [5]. Therefore, in the situation of not having access to remote control, they are limited to using the automation system. Some other previous papers implemented a smart system that users can open the door through their smartphones; However, in our system, an authorized person can enter the key pattern via three embedded switch buttons outside the house to get into the place. Most of the relevant keyless products in the market are in a high price range, so not everyone can afford that system [6]. On the other hand, we extended our project with this feature for less than $\$ 10$, which is affordable for everyone.

\section{System Design and Implementation}

This section details the design and implementation of our proposed IoT-based smart home system for disabled people. Overall, our proposed system has a client-side mobile app and a server-side Raspberry Pi with sensor modules.

\subsection{Android App with Voice Commands}

Considering the accessibility requirement from the disabled people, we choose to provide a voice-based control option on our client-side mobile app. Also given that Android has the largest user groups, we choose to focus on Android on our client-side.

\subsubsection{Android App User Interface}

Android is a popular operating system for mobile devices developed by Google. The core of this operating system is based on Linux. The scope of this operating system is increasing rapidly. Today, Android is used in devices such as smart TVs, smart cars, wearable gadgets, etc. [18]. Raspberry Pi boards are very popular for IoT projects due to their built-in wireless communication protocols. In this Raspberry Pi project, we want to control the GPIO pins on the 
pi board through the Android application, using the Wi-Fi connection [19].

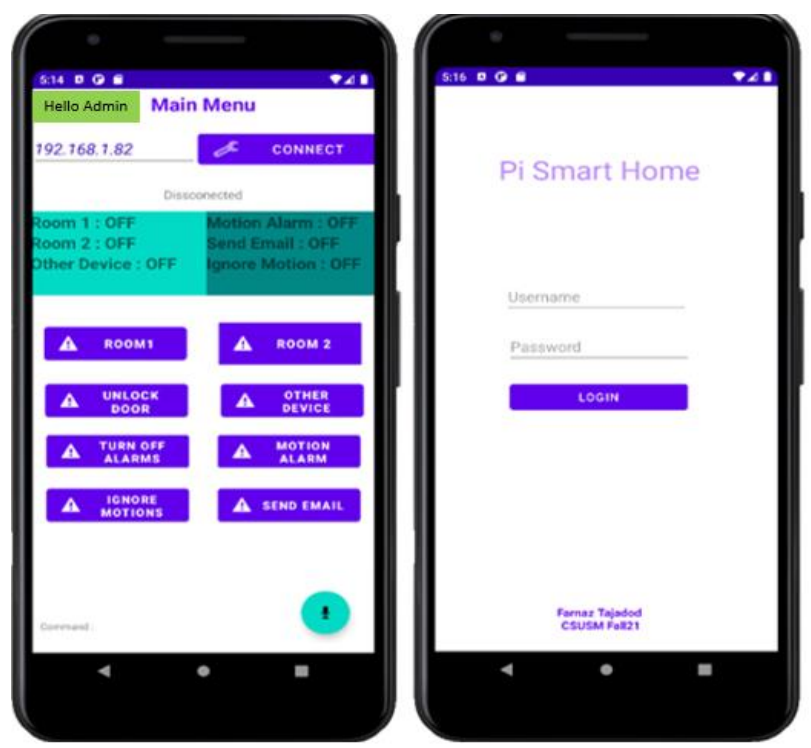

Figure 1 Android App Main Menu and Login Page

In this work, we implemented a remote-control program that allows users to control the smart home system with an Android phone application remotely through the Internet connection. The operation is connected to the Internet by the phone via $\mathrm{Wi}-\mathrm{Fi}$, and then we can use the phone to turn the relays on and off. This program makes the house smarter, and tasks such as controlling the lights, TV, door lock, etc., can be done easier and faster. The Android app for controlling household appliances works so that the corresponding relay is activated by touching each key, and if we touch the key again, the relay turns off. This system uses WI-FI to control devices and lamps with four channels (two 2-channel relays) that can control four different devices separately at the same time.

All home appliances are connected to the Android application on your mobile phone, and then wherever you are in the house, you can see the status of the equipment or command them. For example, you can see which lights are on and turn them off, or you can turn on the TV, or AC. Figure 1 represents the login and the main menu page of the android application.

Eight options are available for users to control the smart home.

1) "Room1": Users can turn on and off the light of room 1 .

2) "Room2": Users can turn on and off the light of room 2.

3) "Unlock Door": Home owners have access to this door controller. They can remotely open the door when authorized people ring the doorbell.
4) "Other Device": Users can turn any other home appliances on and off.

5) "Turn Off Alarms": Users can turn off the alarm by clicking this option when the alert system is triggered due to a wrong unlock pattern or a suspicious motion.

6) "Motion Alarm": If the motion alarm is turned on, it means the security system is activated. If the motion alarm is turned off, it detects motions to turn on lights.

7) "Ignore Motions": Users turn this option on if they have a pet or if they prefer to ignore motions at any time during their presence.

8) "Send Email": If a user activates the send email option, the Admin receives a notification email when a person rings the doorbell (pushes A button), enters an incorrect unlock pattern, or a motion sensor detects any motion.

Users can always review the status of each element in the status panel on the top portion of this interface. They can also switch the status to the previous state by clicking on each option.

There is a listener button on the application's main screen that the user can change the state of sending a command to the listening mode. In Figure 1, the microphone icon can be seen in the lower right corner of the page. When the user clicks on that, Google Assistant will ask: "How can I help you?" and it waits to receive a voice command from a user. We enabled voice recognition to convert all voice commands to text messages and trigger their corresponding action.

\subsubsection{User Access Control}

We consider our proposed smart home system can be controlled by different groups of people. This is a great feature that adds the ability of multi-users to our implementation. Only those people whose login credential has already been registered to the system can use the application, and therefore control the door lock, lights and manage other devices at home. Different users can $\log$ in to the smart system at the same time and control different components. Each user can see the changes made by other users in realtime through the user interface.

We determine three types of users with different control access. This is the most important part of the system in terms of security. We can define each user's access control and permission to control, activate, or change different elements of the system. There are three types of users defined in the system: homeowner, other family members (e.g., kids) and other temporary visitors. When users $\log$ in to the application, the system recognizes their access level and enables the activities based on their access. 
Figure 2 represents the available options for other family members (left) and temporary visitors, e.g., babysitters (right). Besides the "other device" option in the main menu, both users have access to turn on and off room2's light. While other family members can ignore motions, and temporary visitors have access to the door lock. All other grayed-out buttons are unavailable for those users.

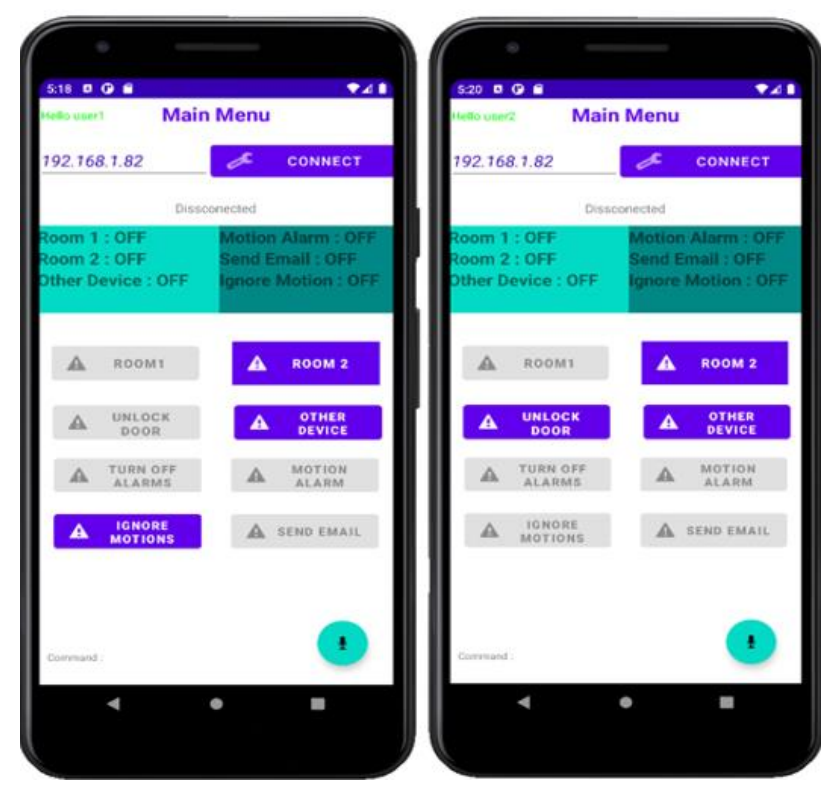

Figure 2 Menu page for different users

Figure 1 gives us the vision of the Admin who has full access to the system and can control and change every element through the application. Admin can deactivate the security mode, review the email notifications, unlock the door for authorized people, and manage the energy consumption by turning off lights and unnecessary devices. User type can be seen in the top left corner of the mobile application screen, which is highlighted in green.

We defined a user permission setter that gets the user type (homeowner, other family members, and temporary visitors) as input. Then it decides which components should be disabled for that specific user by setting the enabled feature of that component to false. For example, if the admin login to the application, nothing gets changed because he has full access to the entire system; However, if other family members login to the application, there are some options such as room1, motion alarm, unlock the door, and send an email that set disabled, shown in Figure 2. Other family members can only control room2's light, toggle another device, and ignore motions which the enabled feature for them has been set to true. Also, we can see from Figure 2 that temporary visitors only have access to turn on/off room2 light, another device, and unluck the door. If a user attempts to $\log$ in to the application with any other username and password not registered into the system, it will get an error message "Wrong username/Password."

In this work, users cannot create their passwords, and different types of users have been already registered to the system. This feature can be considered for the future works so that users can not only choose their username and password, but they can also change the access control by setting the enabled feature to true or false.

\subsubsection{Convenient Voice Commands}

Smart home systems controlled by voice in one of the new smart systems in intelligence building have been used recently. This system has a powerful voice recognition capability. Due to its wide variety of applications, it has been able to open its place in the intelligence industry quickly.

The voice command system in the smart home is made up of two main sections. The first part is related to the voice and voice recognition system. According to the intelligent program, the human voice is detected in this section. If the detected sound according to the protocols and programs matches the previous sound, the person's voice is converted into text, and finally, the command is recognized to the contents [7].

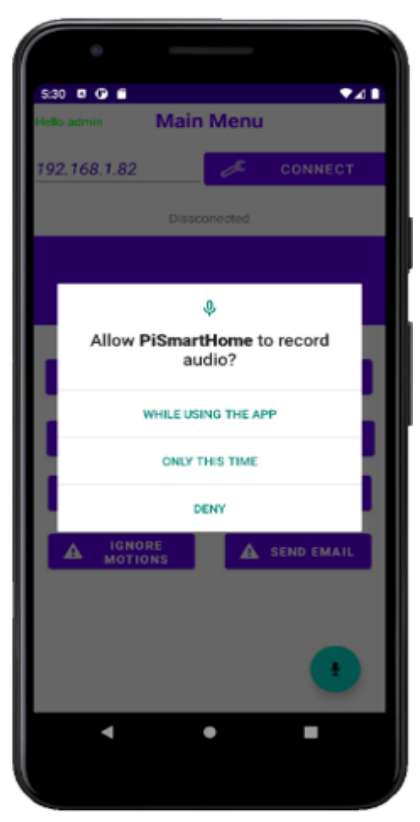

Figure 3: Voice Assistant Permission

The second part of this intelligent voice system includes a wireless system that works as a remote control. It can remotely communicate with other objects in the system, such as lamps, door locks, and 
other devices, and transmit information to that system. Finally, the command is executed by the system [8].

Figure 3 represents a snapshot of the application of our smart home system that asks users for permission to listen and record voice commands through their smartphone's microphone. Users can deny or accept the permission per their preferences.

To integrate the Google Assistant for the android application, we used the Software Development Kit (SDK) that Google has released for android developers to build actions. Google Assistant's speech recognition API gets the voice command and converts it to text through the speech recognizer when a user says a voice command. This text command will be validated and compared with its corresponding socket number to trigger the right action. We defined commands for the listener to get the voice and save it into an array of text. This array finds the best match action for this command.

Table 1: Voice Commands and the Actions

\begin{tabular}{|l|l|c|l|}
\hline \multicolumn{1}{|c|}{ Voice Commands } & \multicolumn{1}{|c|}{ Action } & $\begin{array}{c}\text { Command } \\
\text { code } \\
\text { (Socket) }\end{array}$ & \multicolumn{1}{|c|}{ Details } \\
\hline $\begin{array}{l}\text { Room one, Room 1, } \\
\text { One }\end{array}$ & Toggle Room1 lights & 1011 & $\begin{array}{l}\text { turning on/off Room } \\
\text { one's light }\end{array}$ \\
\hline $\begin{array}{l}\text { Room two, Room 2, } \\
\text { Two }\end{array}$ & Toggle Room2 lights & 2011 & $\begin{array}{l}\text { turning on/off Room } \\
\text { two's light }\end{array}$ \\
\hline Unlock, Door & Unlock the door & 3001 & unlock the door \\
\hline $\begin{array}{l}\text { The device, another } \\
\text { device }\end{array}$ & $\begin{array}{l}\text { Toggle another } \\
\text { device }\end{array}$ & 4011 & $\begin{array}{l}\text { turn on or off other } \\
\text { devices (relay) }\end{array}$ \\
\hline Camera & Show camera & 5001 & show camera screen \\
\hline $\begin{array}{l}\text { Motion Alarm, } \\
\text { Motion }\end{array}$ & $\begin{array}{l}\text { on/off motion } \\
\text { detection }\end{array}$ & 6011 & $\begin{array}{l}\text { use motion sensor } \\
\text { for security system }\end{array}$ \\
\hline $\begin{array}{l}\text { Turn off, Turn off the } \\
\text { alarm }\end{array}$ & $\begin{array}{l}\text { Turn off the noise of } \\
\text { the security alarm }\end{array}$ & 7001 & $\begin{array}{l}\text { Turning off the alarm } \\
\text { system by Admin } \\
\text { only }\end{array}$ \\
\hline Ignore Motion & $\begin{array}{l}\text { Turn off the motion } \\
\text { sensor }\end{array}$ & 8091 & \begin{tabular}{l} 
ignore motion sensor \\
\hline
\end{tabular} \\
\hline
\end{tabular}

Table 1 lists the voice commands that users can use to control the smart home system without touching their phones. This option is an important feature for disabled people who has movement limitation. "Voice Commands" in table lare the words that users can trigger their desired "Actions." The corresponding socket number for each voice command is also represented among more details about the action for each voice command.

Using a voice command system in a smart home has many benefits for disabled users and makes their work easier. If a person uses a wheelchair or cannot use arms, they can easily control the door lock, lights, and any other home appliance by saying a few words. Voice command assistant makes a faster and easier way to use the advantages of our smart system. We trained our smart system with a set of commands (Table 1) that will be converted to text through voice recognition and trigger the related action to respond to users' requests.

\subsection{Raspberry Pi with Sensor Modules}

All the hardware components used in this work are presented in Figure 4 and also listed in Table 2. Apart from a smartphone and a raspberry pi, the most important sensor modules are motion sensor, flame detector, and gas module. These sensors communicate through their signals processing via the breadboard with the Raspberry pi. Sensor modules are the "things" part of an IoT project. Proper configurations need to be set via low-level programming in order to change the behaviors of these devices.

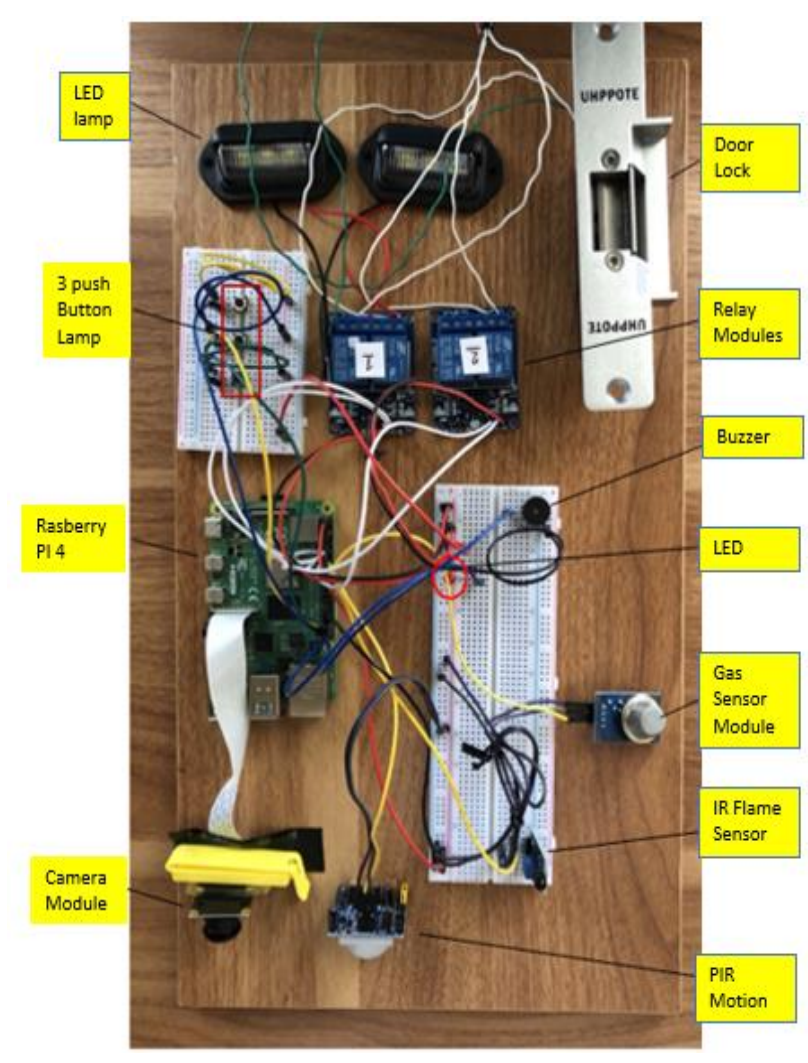

Figure 4 Project Hardware Equipment

\subsubsection{Raspberry Pi}

This system's main piece of hardware equipment is the Raspberry Pi board, an electronic chip that is a small computer that can be programmed and used for various tasks. Raspberry Pi is among the smallest and most powerful computer boards in the world, which in addition to its small size and lightweight, have high speed and accuracy. With the many input and output pins (GPIO pins) on it, we built our new IoT-based Smart Home System [9]. Raspberry Pi in IoT can be used in a wide range of tasks. These boards are ideal for projects requiring a computer, but you do not need much processing and want to keep the costs low and save space. Because Raspberry Pi already has a 
Bluetooth module, we only need to connect it to one device (or multiple devices) and then configure it with a mobile phone to control a particular device from a mobile device.

Table 2 Project Hardware List

\begin{tabular}{|c|c|}
\hline Raspberry Pi 4 & Model B 8 GB Single Board Computer \\
\hline Smartphone & $\begin{array}{l}\text { Xiaomi Redmi } 9 \text { 64GB, 4GB RAM, } \\
\text { 6.53" Full HD + AI Quad Camera, LTE } \\
\text { Factory Unlocked Smartphone }\end{array}$ \\
\hline Camera Module & $\begin{array}{l}\text { LANZO Fisheye Wide Angle 5MP } \\
\text { 1080p Night Vision }\end{array}$ \\
\hline IR Flame Sensor & $\begin{array}{l}\text { Module Detector Smartsense for } \\
\text { Temperature Detection }\end{array}$ \\
\hline Relay Modules & Two SunFounder 2 Channel DC 5V \\
\hline PIR Motion & $\begin{array}{l}\text { HC-SR501 infrared Detector Sensor } \\
\text { Module }\end{array}$ \\
\hline Memory Card & SanDisk 64 GB Ultra Micro SDXC UHS \\
\hline $\begin{array}{l}\text { Push Button } \\
\text { Switch }\end{array}$ & $\begin{array}{l}\text { 5mm Momentary Tactile Tact Push } \\
\text { Button Switch for PCB } 4 \text { Pin DIP Black }\end{array}$ \\
\hline Door Lock & UHPPOTE Electric Strike Fail-Secure \\
\hline $\begin{array}{l}\text { Gas Sensor } \\
\text { Module }\end{array}$ & Detection liquefied \\
\hline Adapter Supply & 12V LED License Plate Light \\
\hline $\begin{array}{l}\text { Raspberry } \mathrm{Pi}_{4} \\
\text { Official PSU }\end{array}$ & DC 5. 1V 3 Amp Power Supply \\
\hline $\begin{array}{l}\text { LED License } \\
\text { Plate Light }\end{array}$ & Voltage: $12 \mathrm{~V}$, power: $1.8 \mathrm{~W}$ \\
\hline Breadboard & $\begin{array}{l}830 \text { Point Proto Shield Distribution } \\
\text { Connecting Blocks }\end{array}$ \\
\hline Breadboard & 400 Point Solderless \\
\hline Buzzer & 5V Active Electronic Alarm Magnetic \\
\hline LED & $\begin{array}{l}\text { Red LED for the alarm system and } \\
\text { motion detector }\end{array}$ \\
\hline $\begin{array}{l}\text { Breadboard } \\
\text { Jumper Wires }\end{array}$ & Dupont Wire (40pin M/F, M/M, F/F) \\
\hline
\end{tabular}

Many previous smart home projects have been implemented by using an Arduino board. Simplicity, easy communications with analog sensors, stability, energy consumption, and reasonable price are some of the advantages of Arduino compared to Raspberry Pi [10]. Considering all these benefits, Arduino may seem to be a better option for sensor-based projects. However, Raspberry Pi can perform multiple tasks at once. For this work, Raspberry Pi can take a picture, send an email, detect Motion, handle several users, detect smoke and hazardous gas, maintenance lights, open door lock, etc. In addition, another advantage of Raspberry Pi is that the operating system [11] can be easily switched on a Raspberry Pi board. A SD card can easily install the operating system on Raspberry $\mathrm{Pi}$, so we can change the operating system by swapping the memory card. Moreover, Raspberry Pi model $\mathrm{B}$ has a network port that can be connected to the local network or the Internet. Even using the USB port, Raspberry Pi can easily connect to the Internet, while connecting an Arduino to the Internet is very difficult. To run an Arduino network, external hardware must be properly connected to it and addressed using code. This small device with very high capabilities is suitable for all complex projects in programming and the Internet of Things. These are the main reasons we decided to use the Raspberry Pi board in this project.

GPIO, or General-Purpose Input /Output, is a feature of most modern embedded computer hardware and a key component of many embedded systems [12]. GPIO pins are connected to the computer circuit board. Users can control the behavior of the GPIO pins by setting them to read data from sensor modules such as motion sensor, gas detector, and flame sensor. Then use the collected data to control different output elements like LEDs and buzzers. The Raspberry Pi 4 Model B has 40 pins that give access to about 25 GPIO lines. These pins have many applications on the Internet of Things and industrial automation [13].

The pins provide the ability to connect Raspberry Pi to other devices and electronic sensors and interact with them. Each pin can be identified by two numbers, named BOARD and BCM (Broadcom SOC channel) numbers. In this work, we used BCM numbers for java programming, which are listed in Table 1. The Standard pins switch in both on and off modes, meaning they only have HIGH and LOW with the 3.3 volts output or input. We used one of the most common functions of GPIO pins which is a configuration in software to be input or output, besides reading the value of digital output.

\subsubsection{Main Sensor Modules}

a) Motion Sensor. The PIR motion detection sensor works with a digital signal, and its sensitivity is adjustable. In this project, when an object detects by the sensor, which defines as the input in the program, a defined output is a LED, and a buzzer will trigger. After detection, the sensor activates, and the LED output turns on, or it starts blinking. There are two applications of the motion sensor in our system, one is to detect Motion to turn on or off lights, and the next is to trigger the alarm.

One of the usages of the motion sensor in this work is that if the user activates the security system by using the interface or through the application, the motion sensor operates as a security system. Therefore, when any motion detects by the PIR module, the buzzer output beeps, and the red LED starts blinking. This sensor also reports the situation when detecting the presence of a burglar or an 
unauthorized person in the home and alerts the users via their smartphone.

Home energy-saving solutions are usually based on occupancy detection to identify the most used points in the house, such as the living room or kitchen, focusing on deploying motion sensors at these points. When the motion sensor detects any occupancy information (occupant vs. empty), it triggers the relay key, and the light of the corresponding room will turn on. The light remains on based on the time we set before truing off again. By adding variables such as brightness, motion sensitivity, and timer, it is possible to use this sensor for different places

Motion sensor can be deactivated, and it is most likely considered for users who have pets and prefer to ignore motions at night so that the lights do not go on and off by detecting any motion. Also, they may prefer to deactivate the security system when they leave to avoid any false alarms due to their pets' movements. There are pet-friendly motion detectors that ignore animals at home. Besides pets, some other elements can trigger false alarms, such as rotating fans and flickering lights.

b) Relay. In this work, we use two 2-Channel Relay modules to electrically operate switches that can turn on or off some elements. One of the relays is used to stimulate keys to turn on/off the room's lights (Room1 and Room2), and the other is to lock/unlock the front door, besides another available key for any home appliance such as TV, AC, etc. The relay module should be powered with $12 \mathrm{~V}$, which is adequate to use with the Raspberry pi. Practically, the most important consideration while working with relay is that this module is triggered (it turns on) when it receives a LOW signal from the raspberry pi. If the relay receives a HIGH signal, it will turn off. In other words, the relay module works with inverted logic.

c) Flame and Gas Detectors. Home safety is not limited to protecting against unauthorized people. Another challenge to home safety is the occurrence of fires which threatens houses with traditional facilities. However, our smart home design has a good solution to this problem. One of our smart home system tools for people with disabilities is the use of environmental sensors. These sensors are equipped to help people with limited movement at home. Smoke and gas sensors are responsible for detecting fires and hazardous gas leakages. In such a situation, the intelligent system acts like a fire brigade. It will alert you with a notification email, activate the alarm system. These sensors are located around the house and can detect fire and toxic gas leakage. In case of any of these incidents, if necessary, the emergency services or the police or via mobile phone will warn. Besides, a family member can easily have accurate monitoring, control, and operation [14].

d) Camera. Individuals with limited physical activity may not react properly when the door is being knocked. They may not feel safe opening the door without knowing the person behind the door. Our Smart Home system has a solution to this issue. When the user with limited movement is alone at home, they can see the picture of the person who rings the doorbell through their mobile phone and decide whether to open the door or not. The camera can be considered the transition of the taken snapshot on the user's smartphone, laptop, or other screens. The fact that the camera information, including images, can be viewed and accessed on the phones via the Internet is a small example of the use of the Internet of Things. This is the connection that is made between objects via the Internet. Commands are sent via a smartphone, and the camera receives and executes these commands [15].

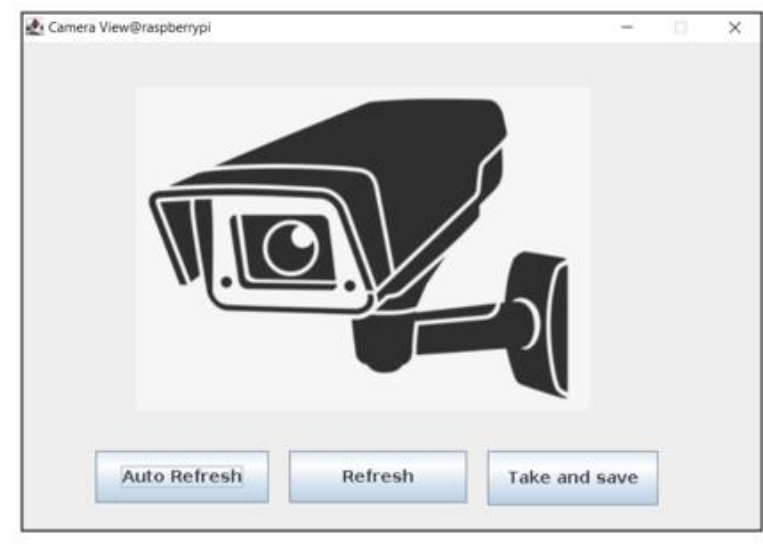

Figure 5 Camera Interface

In our work, two scenarios get the camera activated to take a picture of the one behind the door: First, when someone pushes the "A" button, also considered as the doorbell, in this case, a notification email will be sent to the user includes a picture of that person and a detailed text file contains a date, time and system's activities' information. The second is when a person is trying to unlock the door by entering the incorrect unlock pattern. In this situation, the camera immediately takes a picture and sends an alert notification email to the user to make the user aware that someone is attempting to break into the house. Then the alarm system gets activated, red LED stars blink, and the buzzer beeps. Figure 5 represents the camera Interface screen that users can have access to the camera without any of the above scenarios occurring. This screen contains three options to choose from: Refresh, Auto-refresh, and Take and 
Save. The refresh button is used when the user wants to see only one screenshot of the outside. The camera will take one single shot and represent it to the user. The next option is auto-refresh, which constantly takes a picture every 3 seconds, and the user can see the updated snapshots without refreshing the camera. The last button is the take and saves option, which takes one picture of the front door environment and saves that image for future records. Individuals can use any of these options to activate the camera and capture a picture.

\section{System Evaluation}

To evaluate our proposed system, we consider the door unlock scenario. We implemented an unlock key pattern on the door lock module so that some people such as dog walkers can get into the house with an authorized key pattern. Figure 6 shows that a person rings the doorbell.

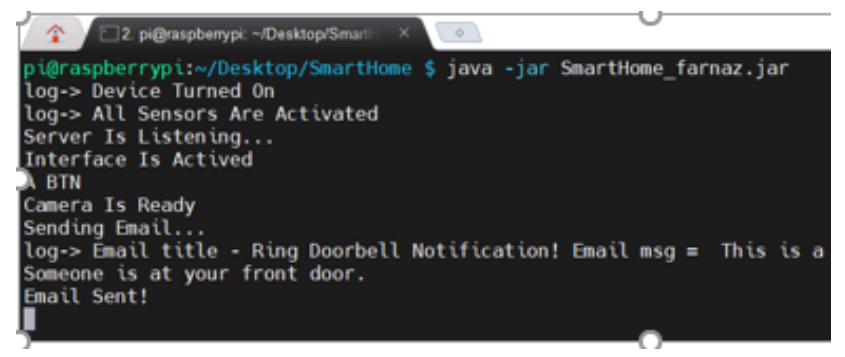

Figure 6 Push the Doorbell (A button)

\section{Smart Home System - Ring Doorbell Notification!}

farnaz@blackrainbow.pro

to me -

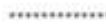

This is a message from your Smart Home System.

Someone is at your front door.

n...n..........

$2021-11-1317-18-43$

2 Attachments
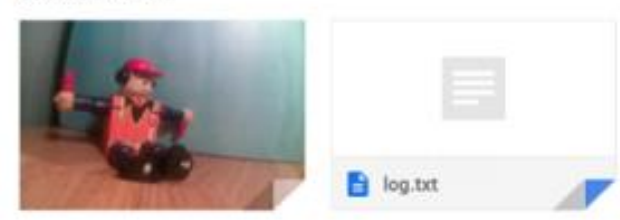

Figure 7 Doorbell Notification Email

Figure 7 is an example of a notification email that a user receives when someone rings the bell. Figure 8 shows a scenario where someone opened the front door by entering the correct unlock pattern. The key pattern for the door lock is defined as "BBAACCBAC"; therefore, any pattern other than this will trigger the security system. If an authorized person enters a wrong pattern, the Admin can still turn off the alert system, ignore the notification email, and even open the door via the application.

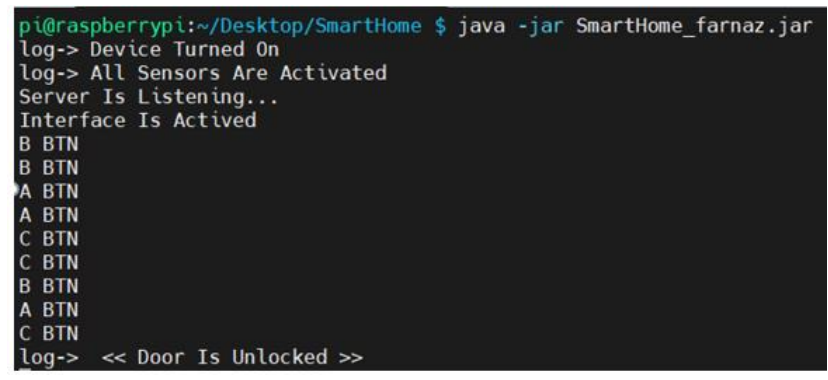

Figure 8 Correct Unlock Pattern

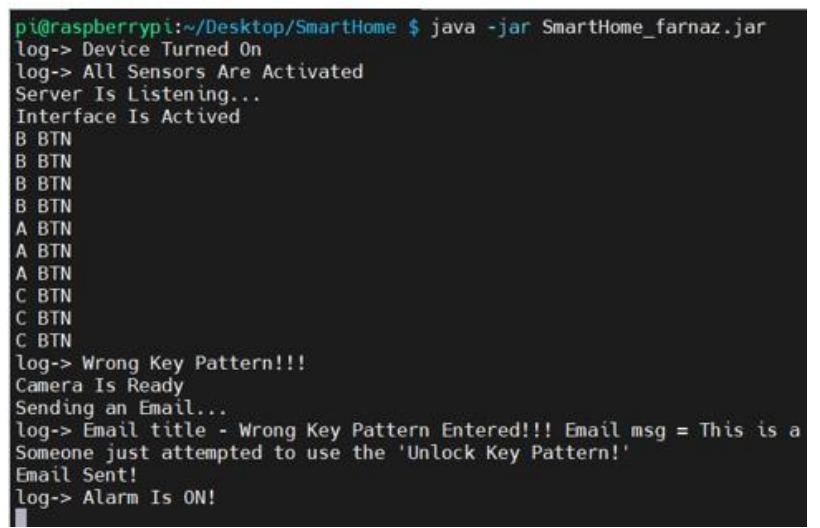

Figure 9 Incorrect Unlock Pattern

Figure 9 represents a scenario where a person enters a random incorrect key pattern. Immediately after the wrong pattern is detected, an alert email with the picture taken from the camera is sent to the homeowner, shown in Figure 10.

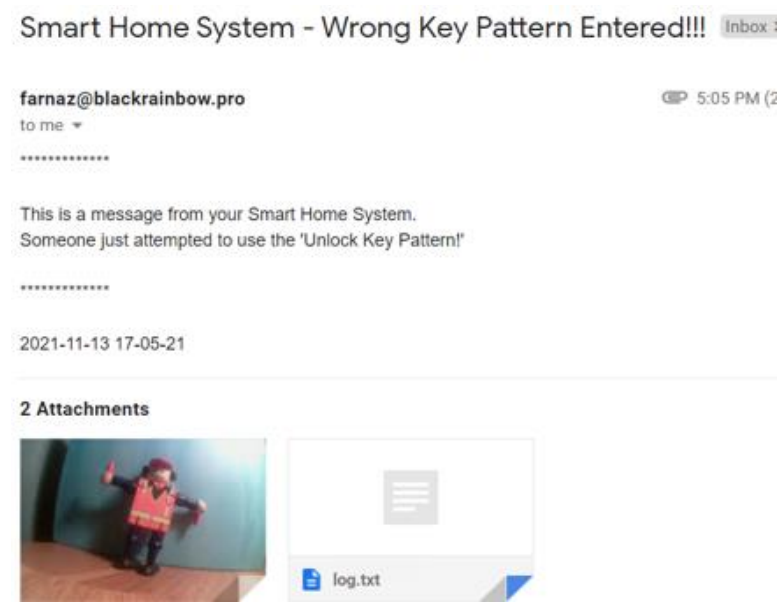

Figure 10 Wrong Key Pattern Alert Email

\section{Conclusion and Future Work}

Future works may consider using a public IP address to control the smart system from anywhere outside the house. Also, the "Key Pattern," which can be entered physically by any authorized person via the 
three buttons (A, B, C), can be replaced by three motion sensors to detect someone's steps combinations, or the user could put their hand over the sensors in a combination. For example, someone approaches the door to unlock it, takes one step to the left, then to the right, step back, and then steps forward. The hand combination would be covered one sensor with the hand and take away, cover the other sensor, and take the hand away, then wave the hand in front of the other sensor. This would unlock the door, and the door will open.

The impact of this IoT-based Smart Home System can improve people's life quality with physical and mental health; Besides, it can bring comfort and relaxation feeling to them. Also, users can easily control and adjust the lighting to reduce costs ultimately. Moreover, there are some objects in this work that distinguish my work from previous studies. Physical key patterns for authorized people, dual motion sensors, user-friendly Android applications, and a real-time interface are some features of this work. Therefore, people with disabilities can easily turn on and off the lights and all kinds of components and devices in the smart home or close the door without having to move, with a simple touch of their smartphones or a voice command. This not only help the disable people but also help their families to constantly watching their disable loved ones from doing tasks around the house [20]. Another feature that can be added to future works is that system automatically calls 911 when any suspicious motion triggers the alarm system or if someone enters the incorrect unlock pattern.

Years ago, we could never imagine that a day would come when we could automatically open doors, turn on/off lights, adjust AC temperature, and many more. Now all this is a fact and not a fantasy! The answer to that is the Internet of Things (IoT); This technology is growing rapidly more than expected. While smart homes undoubtedly offer great benefits to many people with disabilities, smart devices' failure and inaccessibility can be daunting. IoT devices could be placed anywhere, so they may not be plugged into a power outlet, and they could be running off batteries. Therefore, adopting a smart home system to a version that is not dependent on electricity or can use solar power (renewable energy sources) will support a disabled person at a higher level. On the other hand, network connectivity could be a limitation for IoT devices which may not always be continued. There could be certain times when users are not able to connect to the network. If the system needs to be controlled remotely and there is a lack of internet connection, it can cause limitations.

\section{References:}

[1] World Health Organization. Disability and Rehabilitation. Available online: http://www.who.int/disabilities/en/

[2] https://www.theringer.com/movies

[3] Neal-Boylan L., Miller M., and LussierDuynstee P. Failing to Fail When Disability Is a Factor. Nurse Educ. 2021 Jul-Aug 01;46(4):230-233.

[4] Pavithra D, and Balakrishnan R., "IoT based monitoring and control system for home automation," 2015 Global Conference on Communication Technologies (GCCT), 2015.

[5] Gagan: PG scholar, NMAMIT, Nitte, India, "IoT based system for a person with a physical disability" April 2016 2nd Nitte Conference on Advances in Electrical Engineering NCAEE2016. NMAM Institute of Technology, Nitte, Vol. 4, Special Issue 2, April 2016, pp 157 160

[6] Wei Z., and Landay J.A., "Evaluating SpeechBased Smart Devices Using New Usability Heuristics," IEEE Computer Society, pp. 84-96, April-June 2018.

[7] Isyanto H., Arifin A.S., Suryanegara M., "Performance of Smart Personal Assistant Applications Based on Speech Recognition Technology using IoT-based Voice Commands" IEEE Computer Society, DOI: 10.1109/ICTC49870.2020.9289160, Oct.2020.

[8] Chase J. The Best Smart Locks. November 16, 2021.

[9] Stork V., What is Raspberry Pi? Specs and Models (2021 Guide). AUGUST 25, 2021.

[10] https://www.circuitspecialists.com/blog/arduino -vs-raspberry-pi/. Arduino vs Raspberry Pi December 21, 2020.

[11] Traner J., An Introduction to GPIO Programming.

https://www.ics.com/blog/introduction-gpioprogramming. June 12, 2019.

[12] Jesiewcz J., Keans W., Craighead J. Fozard J., Scott S., McCarthy J. Smart rehabilitation for the 21 st century: The Tampa Smart Home for veterans with traumatic brain injury. 2011. ehabilitation and Mental Health Counselling Faculty Publications, Paper 2.

[13] Savage R., Sendula D. Delporte F. and Bug R. The Pi4J project. Java I/O library for the Raspberry Pi. Jan 2021. Version 1.4. 
[14] Midrack R., What Is a Smart Lock and Why Would You Want One? September 10, 2021

[15] MobaXterm dev team. MobaXterm: Enhanced terminal for Windows with X11 server, tabbed SSH client, network tools.

(https://mobaxterm.mobatek.net). New

Releases:

(https://blog.mobatek.net/tags/mobaxterm/).

Mar 2021.

[16] Ismail NH., Tukiran Z., Shamsuddin NN., Saadon E.I.S, "Android-based home door locks application via Bluetooth for disabled people," IEEE Computer Society, DOI: 10.1109, Nov.2014.

[17] Maravitsas N., Example of Android Socket. May 2013.

[18] Isyanto H., Arifin A.S., Suryanegara M., "Design and Implementation of IoT-Based
Smart Home Voice Commands for disabled people using Google Assistant," IEEE

Computer Society, DOI: 10.1109, Feb.2020.

[19] Amdahl G. et al. Special anniversary Editor.

The Newspaper of Information Systems

Management Vol. 26, No. 25, 234 pages. June 1992.

[20] Heath N. What is the Raspberry Pi 4?

Everything you need to know about the tiny, low-cost computer. July 2019.

\section{Creative Commons Attribution License 4.0}

(Attribution 4.0 International, CC BY 4.0)

This article is published under the terms of the Creative Commons Attribution License 4.0

https://creativecommons.org/licenses/by/4.0/deed.en US 